\title{
COVID-19 Antibody Surveillance Among Healthcare Workers in A Non-COVID designated Cardiology Centre
}

\author{
HWEI SUNG LING ${ }^{1}$, Ing Xiang Pang ${ }^{2}$, Alan Yean Yip Fong ${ }^{3}$, Tiong Kiam Ong ${ }^{2}$, Ning \\ Zhang Khiew ${ }^{2}$, Yee Ling $\mathrm{Cham}^{2}$, Asri Said ${ }^{4}$, Yen Yee Oon ${ }^{2}$, Keng Tat $\mathrm{Koh}^{2}$, Chen Ting \\ $\mathrm{Tan}^{2}$, Kian Hui $\mathrm{Ho}^{2}$, Francis Eng Pbeng Shu ${ }^{2}$, Chandan Deepak Bhavnani ${ }^{2}$, and Lean Seng \\ Chen $^{2}$ \\ ${ }^{1}$ Universiti Malaysia Sarawak Faculty of Medicine and Health Sciences \\ ${ }^{2}$ Sarawak Heart Centre \\ ${ }^{3}$ Clinical Research Centre Sarawak \\ ${ }^{4}$ UNIMAS
}

May 15, 2020

\begin{abstract}
BACKGROUND: Reports on healthcare worker antibody response to COVID-19 infection are scarce. We aim to determine the COVID-19 antibody prevalence among healthcare workers in a cardiology centre and the relationship between case definition criteria with the COVID-19 antibody result. METHODS: Convenience sampling was applied. Healthcare workers in Sarawak Heart Centre (SHC) cardiology, radiology, and emergency unit were recruited. A survey form on clinical symptoms and close contact history was distributed. HEALGEN COVID-19 IgG/IgM rapid test was performed using serum/ whole blood specimen. Staff with positive COVID-19 antibody results were referred to the infectious disease specialist for assessment. RESULTS: A total of 310 staff were screened. $220(71 \%)$ were female, and the mean age was $36 \pm 7.7$ years old. $46(14.8 \%)$ staff reported having clinical symptoms at some stage from the end of January 2020 to the time of this surveillance. Number of staff who had a history of overseas travel, close contact with confirmed COVID-19 patients, or had visited places with identified COVID-19 clusters were $4(1.3 \%), 24(7.7 \%)$ and $24(7.7 \%)$ respectively. There were 14 staff $(4.5 \%)$ with positive tests positive, 2 for IgM, and 12 for IgG. All those with positive antibody were subsequently tested negative with RT-PCR test. The history of having clinical symptoms and exposure to COVID-19 cluster area were independently associated with a positive IgG result. CONCLUSION: The application of COVID-19 antibody serology rapid tests could determine true exposure of staff to the infection and allow us to reassess existing measures of infection control within the hospital.
\end{abstract}

TITLE: COVID-19 Antibody Surveillance Among Healthcare Workers in A Non-COVID designated Cardiology Centre

AUTHORS: Hwei Sung Ling ${ }^{1,2}$, Ing Xiang Pang ${ }^{1}$, Lean Seng Chen ${ }^{1}$, Chandan Deepak Bhavnani ${ }^{1}$, Francis Eng Pbeng Shu ${ }^{1}$, Kian Hui Ho ${ }^{1}$, Chen Ting Tan ${ }^{1}$, Keng Tat Koh ${ }^{1}$, Yen Yee Oon ${ }^{1}$, Said Asri ${ }^{1,2}$, Yee Ling Cham $^{1}$, Ning Zhang Khiew ${ }^{1}$, Alan Yean Yip Fong ${ }^{1,3}$, Tiong Kiam Ong ${ }^{1}$

\section{AFFILIATIONS:}

${ }^{1}$ Cardiology Department, Sarawak Heart Centre, Ministry of Health Malaysia

${ }^{2}$ Faculty of Medicine and Health Science, Universiti Malaysia Sarawak

${ }^{3}$ Clinical Research Centre, Ministry of Health Malaysia, Kuching, Sarawak

\section{ACKNOWLEDGMENTS:}


We thank the support from hospital team and various heads of unit in Sarawak Heart Centre that make this surveillance possible. We acknowledge the funds donated by the Persatuan Kakitangan PJS (SHC Staff Society) to purchase the antibody rapid test kits. We thank our hospital's occupational safety team and the infectious disease team of Sarawak General Hospital for all their coordination and support during the surveillance. The authors would like to thank the Director General of Health Malaysia for the permission to publish this paper.

\section{ABSTRACT \\ BACKGROUND:}

Reports on healthcare worker antibody response to COVID-19 infection are scarce. We aim to determine the COVID-19 antibody prevalence among healthcare workers in a cardiology centre and the relationship between case definition criteria with the COVID-19 antibody result.

\section{METHODS:}

Convenience sampling was applied. Healthcare workers in Sarawak Heart Centre (SHC) cardiology, radiology, and emergency unit were recruited. A survey form on clinical symptoms and close contact history was distributed. HEALGEN COVID-19 IgG/IgM rapid test was performed using serum/ whole blood specimen. Staff with positive COVID-19 antibody results were referred to the infectious disease specialist for assessment.

\section{RESULTS:}

A total of 310 staff were screened. 220(71\%) were female, and the mean age was $36 \pm 7.7$ years old. $46(14.8 \%)$ staff reported having clinical symptoms at some stage from the end of January 2020 to the time of this surveillance. Number of staff who had a history of overseas travel, close contact with confirmed COVID19 patients, or had visited places with identified COVID-19 clusters were 4(1.3\%), 24(7.7\%) and 24(7.7\%) respectively. There were 14 staff $(4.5 \%)$ with positive tests positive, 2 for IgM, and 12 for IgG. All those with positive antibody were subsequently tested negative with RT-PCR test. The history of having clinical symptoms and exposure to COVID-19 cluster area were independently associated with a positive IgG result.

\section{CONCLUSION:}

The application of COVID-19 antibody serology rapid tests could determine true exposure of staff to the infection and allow us to reassess existing measures of infection control within the hospital.

Keywords: COVID-19, Healthcare worker, Antibody, Surveillance, Sarawak

\section{Introduction:}

The novel coronavirus disease (COVID-19) or SARS-CoV-2 ${ }^{1}$ gained attention after a citywide lockdown was implemented in Hubei, China. ${ }^{2}$ To date, COVID-19 had infected more than 3,000,000 people in the world. This pandemic has currently left more than 230,000 dead and almost two-thirds of the world's countries locked down. ${ }^{3,4}$

Malaysia recorded its first confirmed COVID case on $25^{\text {th }}$ January $2020 .^{5}$ At the time of writing, Malaysia tallied 6176 confirmed cases and 103 deaths. ${ }^{6}$ Kuching, the most populated city in Sarawak, was among the areas with most confirmed COVID-19 cases and death in Malaysia. ${ }^{7}$ Majority of the cases in Malaysia were contributed by a few clusters, including one religious assembly event in East Malaysia. ${ }^{8}$

SARS-CoV-2 virus displayed high transmissibility $\left(\mathrm{R}_{0} \text { of } 2.68\right)^{9}$ and longer incubation period $(6 \text { days })^{9}$ compared to the Middle East Mediterranean virus. The virus can transmit form human-to-human via respiratory droplets, aerosol, and fecal-oral route. These natures of the virus may explain the rapid spread of the global pandemic and high case-fatality rate. ${ }^{10}$

Healthcare workers are not spared from this disease. ${ }^{11}$ China ${ }^{12}$ recorded $3.47 \%$ and Netherlands $4-9.5 \%$ of infected healthcare workers (HCW). ${ }^{13}$ Contact tracing revealed most of the infected HCW acquired the 